\title{
HÖLDER CONTINUOUS FUNCTIONS AND THEIR ABEL AND LOGARITHMIC MEANS
}

\author{
SUSHIL SHARMA AND S. K. VARMA
}

\begin{abstract}
Mohapatra and Chandra [8] have obtained the degree of approximation for $f \in$ $H_{\alpha}(0 \leq \beta<\alpha \leq 1)$ using infinite matrix $A=\left(a_{n, k}\right)$. Mohapatra and Chandra [7] used Euler, Boral and Taylor means. In the present paper we have obtained the analogous results using Abel $\left(A_{\lambda}\right)$ and Logarithmic $(L)$-means.
\end{abstract}

\section{Introduction}

Let $C_{2 \pi}$ be the Banach space of all $2 \pi$ periodic functions defined on $[-\pi, \pi]$ under the sup norm. For $0<\alpha \leq 1$ and some positive constant $k$.

$$
H_{\alpha}=\left\{f \in C_{2 \pi}:|f(x)-f(y)| \leq k|x-y|^{\alpha}\right\} .
$$

The space $H_{\alpha}$ is a Banach space with the norm $\|\cdot\|_{\alpha}$ defined by

$$
\|f\|_{\alpha}=\|f\|_{c}+\sup _{x, y}\left\{\Delta^{\alpha} f(x, y)\right\}
$$

where

$$
\|f\|_{c}=\sup _{-\pi \leq x \leq \pi}|f(x)|
$$

and

$$
\Delta^{\alpha} f(x, y)=\frac{|f(x)-f(y)|}{|x-y|^{\alpha}} \quad(x \neq y)
$$

we shall use the convention that $\Delta^{0} f(x, y)=0$. The elements of the space $H_{\alpha}$ are called Hölder continuous functions. If $D$ is the collection of all differentiable functions defined on $[-\pi, \pi]$ then it is easy to see that

$$
C_{2 \pi} \supseteq H_{\beta} \supseteq H_{\alpha} \supset D \quad \text { for } \quad 0 \leq \beta \leq \alpha \leq 1 .
$$

For each $f \in H_{\alpha}, 0<\alpha \leq 1$, let the Fourier series be given by

$$
f(x) \sim \frac{a_{0}}{2}+\sum_{v=1}^{\infty}\left(a_{v} \cos v x+b_{v} \sin v x\right)
$$

Received June 20, 1997; revised March 3, 1999.

1991 Mathematics Subject Classification. 42A10, 41A25

Key words and phrases. Approximation, Abel mean, Logarithmic mean. 
where $a_{v}$ and $b_{v}$ are Fourier coefficients.

Given $\lambda>-1$, we say that the series $\sum U_{m}$ is $A_{\lambda}$-summable to a finite sum $S$ if the series

$$
(1-r)^{\lambda+1} \sum_{n=0}^{\infty} \epsilon_{n}^{\lambda} r^{n} S_{n}
$$

is convergent for all $r$ in $(0,1)$ and tends to $S$ as $r \rightarrow 1$ in $(0,1)$, where

$$
e_{n}^{\lambda}=\left(\begin{array}{c}
n+\lambda \\
n
\end{array}\right) \quad \text { and } \quad S_{n}=\sum_{m=0}^{n} U_{m} .
$$

If $\lambda=0, A_{\lambda}$-method reduces to well known Abel method of summability (Hardy [3]).

Borwein [1] introduced Logarithmic $(L)$-method of summabililty. He defined a series $\sum U_{m}$ to be summable by $L$-method of summability to the sum $S$ if, for $r$ in the interval $(0,1)$

$$
\lim _{r \rightarrow 1-0} \frac{1}{|\log (1-r)|} \sum_{n=1}^{\infty} S_{n} \frac{r^{n}}{n}=S .
$$

We have following inclusion relation

$$
(L) \supset(A, \lambda) \supset A \supset(C, \delta) \text { for every } \delta>-1 .
$$

We shall use the following notations throughout this paper

$$
\begin{aligned}
2 \phi_{x}(t) & =f(x+t)+f(x-t)-2 f(x), \\
A_{\lambda}(r, t) & =(1-r)^{\lambda+1} \sum_{n=0}^{\infty} e_{n}^{\lambda} r^{n} \frac{\sin \left(n+\frac{1}{2}\right) t}{\sin \frac{t}{2}} \\
L(r, t) & =\frac{1}{t} \tan ^{-1}\left(\frac{r \sin t}{1-r \cos t}\right) .
\end{aligned}
$$

\section{Statement of Results}

Khan [5] obtained the necessary and sufficient condition for $A_{\lambda}$-summability of Fourier series (1.4) and Hsiang [4] obtained the necessary and sufficient condition for $L$-summability of Fourier series (1.4).

Prossdorf [10] proved the following theorem $A$ with a view to obtain the degree of convergence of the Fejér means of the Fourier series of $f \in H_{\alpha}$.

Theorem A. Let $f \in H_{\alpha}, 0<\alpha \leq 1$ and $0 \leq \beta<\alpha$, then

$$
\left\|\sigma_{n}(f: x)-f\right\|_{\beta}=\left[\begin{array}{ll}
O\left(n^{\beta-\alpha}\right), & 0<\alpha<1, \\
O\left\{n^{\beta-1}(1+\log n)^{1-\beta}\right\}, \alpha=1 .
\end{array}\right.
$$


Mohapatra and Chandra [7] replaced the Fejér means of the series (1.4) by well known Nörlund and $\left(\bar{N}, p_{n}\right)$ means. Mohapatra and Chandra [8] replaced Nörlund and $\left(\bar{N}, p_{n}\right)$ by a more general infinite matrix $T=\left(a_{n, k}\right)$ with some conditions on $\left(a_{n, k}\right)$. Mohapatra [9] proved results on degree of approximation of Hölder continuous functions. Das, Ojha and Ray [2] used Borel means to obtain the degree of approximation. Mohapatra and Chandra [7] used Euler, Boral and Taylor means to obtain the degree of approximation for $f \in H_{\alpha}$. They proved the following theorem:

Theorem B. Let $0 \leq \beta<\alpha \leq 1$. Then, for $f \in H_{\alpha}$,

$$
\left\|B^{r}(f: x)-f\right\|_{\beta}=O\left\{r^{-(1 / 2)(\alpha-\beta)}(\log r)^{\beta / \alpha}\right\}
$$

where $B^{r}(f: x)$ is the Boral mean of the series (1.4).

In the present paper we have used $A_{\lambda}$-mean and $L$-mean. In fact we shall prove the following theorems:

Theorem 1. Let $0 \leq \beta<\alpha \leq 1$. Then, for $f \in H_{\alpha}$

$$
\left\|A_{\lambda}^{r}(f)-f\right\|_{\beta}=O\left[(1-r)^{\alpha-\beta}\right]
$$

where $A_{\lambda}^{r}(f)$ is the $A_{\lambda}$-mean of the series (1.4).

Theorem 2. Let $0 \leq \beta<\alpha \leq 1$. Then for $f \in H_{\alpha}$

$$
\left\|L^{r}(f)-f\right\|_{\beta}=O\left[\log (1-r)^{(\beta / \alpha)-1}(1-r)^{\alpha-\beta} r^{\beta}\right]
$$

where $L^{r}(f)$ is the L-mean of the series (1.4).

\section{Preliminary Lemmas}

We shall use the following lemmas for the proof of our theorems.

Lemma 1. If $f \in H_{\alpha}(0<\alpha \leq 1)$ then

and

$$
\left|\phi_{x}(t)-\phi_{y}(t)\right| \leq 4 k|x-y|^{\alpha}
$$

$$
\left|\phi_{x}(t)-\phi_{y}(t)\right| \leq 4 k|t|^{\alpha}
$$

where $k$ is a positive constant. The proof of the lemma is obvious from the definition of $\phi_{x}(t)$ and the function space $H_{\alpha}$.

Lemma 2. Khan [6]

$$
A_{\lambda}(r, t)=\left[\begin{array}{rl}
O\left[\left(\frac{1}{1-r}\right)\right], & \text { when } t \leq 1-r \\
0 & <t \leq \pi \\
0 & \leq r<1 \\
O\left[\frac{(1-r)^{\lambda+1}}{t^{\lambda+2}}\right], \text { when } t & \geq 1-r \\
0 & <t \leq \pi \\
0 & \leq r<1 .
\end{array}\right.
$$


Lemma 3. Hsiang [3] For $0<r<1$,

$$
\frac{1}{t} \tan ^{-1}\left(\frac{r \sin t}{1-r \cos t}\right)=\left[\begin{array}{l}
O\left[\frac{r}{1-r}\right], 0<t \leq 1-r \\
O\left[\frac{1}{t}\right], \quad 1-r<t \leq \pi .
\end{array}\right.
$$

\section{Proof of The Theorem 1}

Let

$$
\begin{aligned}
I_{n}(x) & =A_{\lambda}^{r}(f, x)-f(x) \\
& =(1-r)^{\lambda+1} \sum_{n=0}^{\infty}\left[S_{n}(x)-f(x)\right] r^{n} \epsilon_{n}^{\lambda} \\
& =\frac{(1-r)^{\lambda+1}}{\pi} \sum_{n=0}^{\infty} r^{n} \epsilon_{n}^{\lambda} \int_{0}^{\pi} \frac{\phi_{x}(t) \sin (n+1 / 2) t}{\sin t / 2} d t .
\end{aligned}
$$

Therefore

$$
\begin{aligned}
I_{n}(x)-I_{n}(y) & =\frac{1}{\pi} \int_{0}^{\pi}\left|\phi_{x}(t)-\phi_{y}(t)\right| A_{\lambda}(r, t) d t \\
& =\frac{1}{\pi}\left[\int_{0}^{1-r}+\int_{1-r}^{\pi}\right]\left|\phi_{x}(t)-\phi_{y}(t)\right| A_{\lambda}(r, t) d t \\
& =I_{1}+I_{2}, \text { say. }
\end{aligned}
$$

So

$$
\begin{aligned}
I_{1} & =\frac{1}{\pi} \int_{0}^{1-r} t^{\alpha}\left(\frac{1}{1-r}\right) d t, \text { by }(3.2) \text { and }(3.3) . \\
& =O(1-r)^{\alpha} .
\end{aligned}
$$

and

$$
\begin{aligned}
I_{2} & =\frac{1}{\pi} \int_{1-r}^{\pi}(1-r)^{\lambda+1} t^{\alpha-\lambda-2} d t ; \text { by }(3.2) \text { and }(3.3) \\
& =O(1-r)^{\alpha} .
\end{aligned}
$$

Similarly if we use (3.1) in place of (3.2), we get

$$
\begin{aligned}
I_{1} & =O(1) \int_{0}^{1-r} \frac{|x-y|^{\alpha}}{(1-r)} d t \\
& =O|x-y|^{\alpha} .
\end{aligned}
$$

and 


$$
\begin{aligned}
I_{2} & =O(1) \int_{1-r}^{\pi} \frac{|x-y|^{\alpha}}{t^{\lambda+2}}(1-r)^{\lambda+1} d t \\
& =O|x-y|^{\alpha} .
\end{aligned}
$$

Now for $k=1,2$, we observe that

$$
I_{k}=I_{k}^{1-\beta / \alpha} I_{k}^{\beta / \alpha}
$$

So by (4.2), (4.3), (4.4) and (4.5), we have

$$
I_{1}=O\left\{|x-y|^{\beta}(1-r)^{\alpha-\beta}\right\}
$$

and

$$
I_{2}=O\left\{|x-y|^{\beta}(1-r)^{\alpha-\beta}\right\}
$$

So

$$
\sup _{\substack{x, y \\ x \neq y}}\left|\Delta^{\beta} I_{n}(x, y)\right|=O\left\{(1-r)^{\alpha-\beta}\right\} .
$$

This completes the proof of the Theorem 1 .

Proof of theorem 2.

$$
I_{n}(x)=\frac{1}{|\log (1-r)|} \frac{1}{\pi} \int_{0}^{\pi} \frac{\phi_{x}(t)}{\sin t / 2} \sum_{n=1}^{\infty} \frac{r^{n}}{n} \sin (n+1 / 2) t d t .
$$

So

$$
\begin{aligned}
I_{n}(x)-I_{n}(y) & =\frac{1}{|\log (1-r)|} \frac{1}{\pi} \int_{0}^{\pi} \frac{\phi_{x}(t)-\phi_{y}(t)}{\sin t / 2} \sum_{n=1}^{\infty} r^{n} \sin (n+1 / 2) t d t \\
& =\frac{1}{|\log (1-r)|} \frac{1}{\pi}\left[\int_{0}^{1-r}+\int_{1-r}^{\pi}\right] \frac{\left|\phi_{x}(t)-\phi_{y}(t)\right|}{\sin t / 2} \sum_{n=1}^{\infty} r^{n} \cdot \sin (n+1 / 2) t d t \\
& =I_{1}+I_{2}, \text { say } \\
I_{1}= & \frac{1}{|\log (1-r)|} O(1) \int_{0}^{1-r} \frac{r}{(1-r)} t^{\alpha} d t, \text { by using }(3.2) \text { and }(3.4) \\
= & O\left(\frac{r}{|\log (1-r)|}(1-r)^{\alpha}\right) \\
I_{2}= & \frac{1}{|\log (1-r)|} \frac{1}{\pi} \int_{1-r}^{\pi} \frac{\left|\phi_{x}(t)-\phi_{y}(t)\right|}{\sin t / 2} \sum_{n=1}^{\infty} r^{n} \sin (n+1 / 2) t d t \\
= & O(1) \frac{1}{|\log (1-r)|} \int_{1-r}^{\pi} t^{\alpha-1} d t, \text { by using }(3.2) \text { and }(3.4) \\
= & O\left\{\frac{(1-r)^{\alpha}}{|\log (1-r)|}\right\}
\end{aligned}
$$


Similarly if we use (3.1) in place of (3.2), we get

$$
\begin{aligned}
I_{1} & =\frac{O(1)}{|\log (1-r)|} \int_{0}^{1-r}|x-y|^{\alpha}\left(\frac{r}{1-r}\right) d t \\
& =O\left(\frac{|x-y|^{\alpha} r}{|\log (1-r)|}\right)
\end{aligned}
$$

and

$$
\begin{aligned}
I_{2} & =\frac{O(1)}{|\log (1-r)|} \int_{1-r}^{\pi} \frac{|x-y|^{\alpha}}{t} d t \\
& =O\left\{|x-y|^{\alpha}\right\}
\end{aligned}
$$

Now for $k=1,2$ we observe that

$$
I_{k}=I_{k}^{1-(\beta / \alpha)} I_{k}^{\beta / \alpha}
$$

So by (4.7), (4.8), (4.9) and (4.10), we have

$$
\begin{aligned}
I_{1} & =O\left[\frac{|x-y|^{\beta} r^{\beta / \alpha} r^{(\alpha-\beta) / \alpha}(1-r)^{\alpha-\beta}}{\log (1-r)}\right] \\
& =O\left[\frac{|x-y|^{\beta} r^{\beta}(1-r)^{\alpha-\beta}}{\log (1-r)}\right] \\
I_{2} & =O\left[\frac{|x-y|^{\beta}\{\log (1-r)\}^{\beta / \alpha}(1-r)^{\alpha-\beta}}{|\log (1-r)|}\right]
\end{aligned}
$$

Since $\beta<1$ and $r \leq 1,(4.11)$ and (4.12) gives

$$
\sup _{\substack{x, y \\ x \neq y}}\left|\Delta^{\beta} I_{n}(x, y)\right|=O\left[\{\log (1-r)\}^{(\beta / \alpha)-1}(1-r)^{\alpha-\beta} r^{\beta}\right]
$$

This completes the proof of the Theorem 2 .

\section{Acknowledgement}

Authors extend thanks to Professor Prem Chandra for this paper.

\section{References}

[1] D. Borwein, A logarithmic method of summability, J. Lond. Math. Soc. 33(1958), 212-220.

[2] G. Das, A. K. Ojha and B. K. Ray, Degree of approximation of functions associated with Hardy-Littlewood series in the Hölder metric by Boral means, J. Math. Anal. Appl. 219(1998), 279-293.

[3] G. H. Hardy, Divergent Series, Oxford Publication, 1949.

[4] F. C. Hsiang, Summability of the Fourier series, Bull. Amer. Math. Soc. 67(1961), 150-153. 
[5] H. Khan, On some aspects of summability, Ind. J. pure appl. Math. 6(19.75), 1468-1472.

[6] H. Khan, On the degree of approximation, Math. Chronicle 10(1981); 63-72.

[7] R. N. Mohapatra and.P. Chandra, Hölder continuous functions and their Euler, Boral and Taylor means, Math. Chronicle 11(1982), 81-96.

[8] R. N. Mohapatra and P. Chandra, Degree of approximation of functions in the Hölder matrix, Acta Math. Hung. 41(1983), 67-76.

[9] R. N. Mohapatra, Degree of approximation of Hölder continuous functions, Math. Nachr. 140(1988), 91-96.

[10] S. Prossdorf, Zur Konvergenz der Fourierreihen Hölderstetiger Funktionen, Math. Nachr., 6.9(1975), 7-14.

Department of Mathematics, Madhav Science College, Ujjain, M. P., India.

Department of Mathematics, Govt. Autonomous Science Post-Graduate College, Bilaspur, M. P., India. 